\title{
OPTIMIZATION THROUGH BEST PROXIMITY POINTS FOR MULTIVALUED $F$-CONTRACTIONS
}

\author{
PRADIP DEBNATH
}

Received 18 May, 2020

\begin{abstract}
Best proximity point theorems ensure the existence of an approximate optimal solution to the equations of the type $f(x)=x$ when $f$ is not a self-map and a solution of the same does not necessarily exist. Best proximity points theorems, therefore, serve as a powerful tool in the theory of optimization and approximation. The aim of this article is to consider a global optimization problem in the context of best proximity points in a complete metric space. We establish an existence of best proximity result for multivalued mappings using Wardowski's technique.
\end{abstract}

2010 Mathematics Subject Classification: 47H10; 54H25; 54E50

Keywords: best proximity point, fixed point, $F$-contraction, complete metric space, multivalued map, optimization

\section{INTRODUCTION AND PRELIMINARIES}

Nadler [9] defined a Hausdorff concept by considering the distance between two arbitrary sets as follows.

Let $(\Omega, \eta)$ be a complete metric space (in short, MS) and let $C B(\Omega)$ be the family of all nonempty closed and bounded subsets of the nonempty set $\Omega$. For $\mathcal{M}, \mathcal{N} \in$ $C B(\Omega)$, define the map $\mathcal{H}: C B(\Omega) \times C B(\Omega) \rightarrow[0, \infty)$ by

$$
\mathcal{H}(\mathcal{M}, \mathcal{N})=\max \left\{\sup _{\xi \in \mathcal{N}} \Delta(\xi, \mathcal{M}), \sup _{\delta \in \mathcal{M}} \Delta(\delta, \mathcal{N})\right\},
$$

where $\Delta(\delta, \mathcal{N})=\inf _{\xi \in \mathcal{K}} \eta(\delta, \xi)$. Then $(C B(\Omega), \mathcal{H})$ is an MS induced by $\eta$.

Let $\mathcal{M}, \mathcal{N}$ be any two nonempty subsets of the $\operatorname{MS}(\Omega, \eta)$. The following notations will be used throughout:

$$
\begin{aligned}
& \mathcal{M}_{0}=\{\mu \in \mathcal{M}: \eta(\mu, v)=\eta(\mathcal{M}, \mathcal{N}) \text { for some } v \in \mathcal{N}\}, \\
& \mathcal{N}_{0}=\{v \in \mathcal{N}: \eta(\mu, v)=\eta(\mathscr{M}, \mathcal{N}) \text { for some } \mu \in \mathcal{M}\},
\end{aligned}
$$

where $\eta(\mathcal{M}, \mathcal{N})=\inf \{\eta(\mu, v): \mu \in \mathcal{M}, \nu \in \mathcal{N}\}$.

This research is supported by UGC (Ministry of HRD, Govt. of India) through UGC-BSR Start-Up Grant vide letter No. F.30-452/2018(BSR) dated 12 Feb 2019. 
For $\mathcal{M}, \mathcal{N} \in C B(\Omega)$, we have

$$
\eta(\mathcal{M}, \mathcal{N}) \leq \mathcal{H}(\mathcal{M}, \mathcal{N}) .
$$

We say that $\mu \in \mathcal{M}$ is a best proximity point (in short, BPP) of the multivalued map $\Gamma: \mathcal{M} \rightarrow C B(\mathcal{N})$ if $\Delta(\mu, \Gamma \mu)=\eta(\mathcal{M}, \mathcal{N}) . v \in \Omega$ is said to be a fixed point of the multivalued map $\Gamma: \Omega \rightarrow C B(\Omega)$ if $v \in \Gamma v$.

\section{Remark 1.}

(1) In the MS $(C B(\Omega), \mathcal{H}), v \in \Omega$ is a fixed point of $\Gamma$ if and only if $\Delta(v, \Gamma v)=0$.

(2) If $\eta(\mathcal{M}, \mathcal{N})=0$, then a fixed point and a BPP are identical.

(3) The metric function $\eta: \Omega \times \Omega \rightarrow[0, \infty)$ is continuous in the sense that if $\left\{v_{n}\right\},\left\{\xi_{n}\right\}$ are two sequences in $\Omega$ with $\left(v_{n}, \xi_{n}\right) \rightarrow(v, \xi)$ for some $v, \xi \in \Omega$, as $n \rightarrow \infty$, then $\eta\left(v_{n}, \xi_{n}\right) \rightarrow \eta(v, \xi)$ as $n \rightarrow \infty$. The function $\Delta$ is continuous in the sense that if $v_{n} \rightarrow v$ as $n \rightarrow \infty$, then $\Delta\left(v_{n}, \mathcal{M}\right) \rightarrow \Delta(v, \mathcal{M})$ as $n \rightarrow \infty$ for any $\mathcal{M} \subseteq \Omega$.

The following Lemmas are noteworthy.

Lemma $1([2,4])$. Let $(\Omega, \eta)$ be an $M S$ and $\mathcal{M}, \mathcal{N} \in C B(\Omega)$. Then

(1) $\Delta(\mu, \mathcal{N}) \leq \eta(\mu, \gamma)$ for any $\gamma \in \mathcal{N}$ and $\mu \in \Omega$;

(2) $\Delta(\mu, \mathcal{N}) \leq \mathcal{H}(\mathcal{M}, \mathcal{N})$ for any $\mu \in \mathcal{M}$.

Lemma 2 ([9]). Let $\mathcal{M}, \mathcal{N} \in C B(\Omega)$ and let $v \in \mathcal{M}$, then for any $r>0$, there exists $\xi \in \mathcal{N}$ such that

$$
\eta(v, \xi) \leq \mathcal{H}(\mathcal{M}, \mathcal{N})+r
$$

But we may not have any $\xi \in \mathcal{N}$ such that

$$
\eta(v, \xi) \leq \mathcal{H}(\mathcal{M}, \mathcal{N}) .
$$

Further, when $\mathcal{N}$ is compact, there exists $\xi \in \Omega$ such that $\eta(v, \xi) \leq \mathcal{H}(\mathcal{M}, \mathcal{N})$.

The concept of $\mathcal{H}$-continuity for multivalued maps is listed next.

Definition 1 ([5]). Let $(\Omega, \eta)$ be an MS. We say that a multivalued map $\Gamma: \Omega \rightarrow$ $C B(\Omega)$ is $\mathcal{H}$-continuous at a point $\mu_{0}$, if for each sequence $\left\{\mu_{n}\right\} \subset \Omega$, such that $\lim _{n \rightarrow \infty} \eta\left(\mu_{n}, \mu_{0}\right)=0$, we have $\lim _{n \rightarrow \infty} \mathcal{H}\left(\Gamma \mu_{n}, \Gamma \mu_{0}\right)=0$ (i.e., if $\mu_{n} \rightarrow \mu_{0}$, then $\Gamma \mu_{n} \rightarrow \Gamma \mu_{0}$ as $n \rightarrow \infty)$.

Definition 2 ([9]). Let $\Gamma: \Omega \rightarrow C B(\Omega)$ be a multivalued map. We say that $\Gamma$ is a multivalued contraction if $\mathcal{H}(\Gamma \mu, \Gamma v) \leq \lambda \eta(\mu, v)$ for all $\mu, v \in \Omega$, where $\lambda \in[0,1)$.

\section{Remark 2.}

(1) If $\Gamma$ is $\mathcal{H}$-continuous on every point of $\mathcal{M} \subseteq \Omega$, then it is said to be continuous on $\mathcal{M}$.

(2) A multivalued contraction $\Gamma$ is $\mathcal{H}$-continuous.

In 2012, Wardowski [16] defined the concept of $F$-contraction as follows. 
Definition 3. Let $F:(0,+\infty) \rightarrow(-\infty,+\infty)$ be a function which satisfies the following:

(F1) $F$ is strictly increasing;

(F2) For each sequence $\left\{u_{n}\right\}_{n \in \mathbb{N}} \subset(0,+\infty)$,

$$
\lim _{n \rightarrow+\infty} u_{n}=0 \text { if and only if } \lim _{n \rightarrow+\infty} F\left(u_{n}\right)=-\infty ;
$$

(F3) There is $t \in(0,1)$ such that $\lim _{u \rightarrow 0^{+}} u^{t} F(u)=0$.

Let $\mathcal{F}$ denote the class of all such functions $F$. If $(\Omega, \eta)$ is an MS, then a self-map $T: \Omega \rightarrow \Omega$ is said to be an $F$-contraction if there exist $\tau>0, F \in \mathcal{F}$, such that for all $\mu, \nu \in \Omega$,

$$
\eta(T \mu, T \nu)>0 \Rightarrow \tau+F(\eta(T \mu, T \nu)) \leq F(\eta(\mu, \nu)) .
$$

Multivalued $F$-contractions were defined by Altun et al. [1] as follows.

Definition 4 ([1]). Let $(\Omega, \eta)$ be an MS. A multivalued map $\Gamma: \Omega \rightarrow C B(\Omega)$ is said to be a multivalued $F$-contraction (MVFC, in short) if there exist $\tau>0$ and $F \in \mathcal{F}$ such that

$$
\tau+F(\mathcal{H}(\Gamma \mu, \Gamma v)) \leq F(\eta(\mu, v))
$$

for all $\mu, \nu \in \Omega$ with $\Gamma \mu \neq \Gamma$ v.

Remark 3. An MVFC is $\mathcal{H}$-continuous.

We can find the concept of $P$-property in [12], whereas the notion of weak $P$ property was defined by Zhang et al. [18].

Definition 5 ([12]). Let $(\Omega, \eta)$ be an MS and $\mathcal{M}, \mathcal{N}$ be two non-empty subsets of $\Omega$ such that $\mathcal{M}_{0} \neq \phi$. The pair $(\mathcal{M}, \mathcal{N})$ is said to have the $P$-property if and only if $\eta\left(\mu_{1}, v_{1}\right)=\eta(\mathcal{M}, \mathcal{N})=\eta\left(\mu_{2}, v_{2}\right)$ implies $\eta\left(\mu_{1}, \mu_{2}\right)=\eta\left(v_{1}, v_{2}\right)$, where $\mu_{1}, \mu_{2} \in \mathcal{M}_{0}$ and $v_{1}, v_{2} \in \mathcal{N}$.

Definition 6 ([18]). Let $(\Omega, \eta)$ be an MS and $\mathcal{M}, \mathcal{N}$ be two non-empty subsets of $\Omega$ such that $\mathcal{M}_{0} \neq \phi$. The pair $(\mathcal{M}, \mathcal{N})$ is said to have the weak $P$-property if and only if $\eta\left(\mu_{1}, v_{1}\right)=\eta(\mathcal{M}, \mathcal{N})=\eta\left(\mu_{2}, \nu_{2}\right)$ implies $\eta\left(\mu_{1}, \mu_{2}\right) \leq \eta\left(v_{1}, \nu_{2}\right)$, where $\mu_{1}, \mu_{2} \in \mathcal{M}_{0}$ and $v_{1}, v_{2} \in \mathcal{N}_{0}$.

BPP theorems for $F$-contractive non-self mappings were studied by Omidvari et al. [11] with the help of $P$-property. Later, Nazari [10] investigated BPPs for a particular type of generalized multivalued contractions by using the weak $P$-property.

Srivastava et al. [13,14] presented Krasnosel'skii type hybrid fixed point theorems and found their very interesting applications to fractional integral equations. Xu et al. [17] proved Schwarz lemma that involves boundary fixed point. Very recently, Debnath and Srivastava [6] investigated common BPPs for multivalued contractive pairs of mappings in connection with global optimization. Debnath and Srivastava [7] also proved new extensions of Kannan's and Reich's theorems in the context 
of multivalued mappings using Wardowski's technique. Further, a very significant application of fixed points of $F(\psi, \varphi)$-contractions to fractional differential equations was recently provided by Srivastava et al. [15].

In this paper, we introduce a best proximity result for multivalued mappings with the help of $F$-contraction and the weak $P$ property. Also we provide an example where the $P$-property is not satisfied but the weak $P$-property holds.

\section{BEST PROXIMITY POINT FOR MVFC}

In this section, with the help of the notion of $F$-contraction, we show that an MVFC satisfying certain conditions admits a BPP.

Theorem 1. Let $(\Omega, \eta)$ be a complete $M S$ and $\mathcal{M}, \mathcal{N}$ be two non-empty closed subsets of $\Omega$ such that $\mathcal{M}_{0} \neq \phi$ and that the pair $(\mathcal{M}, \mathcal{N})$ has the weak P-property. Suppose $\Gamma: \mathcal{M} \rightarrow C B(\mathcal{N})$ be a MVFC such that $\Gamma \mu$ is compact for each $\mu \in \mathcal{M}$ and $\Gamma \mu \subseteq \mathcal{N}_{0}$ for all $\mu \in \mathcal{M}_{0}$. Then $\Gamma$ has a BPP.

Proof. Fix $\mu_{0} \in \mathcal{M}_{0}$ and choose $v_{0} \in \Gamma \mu_{0} \subseteq \mathcal{N}_{0}$. By the definition of $\mathcal{N}_{0}$, we can select $\mu_{1} \in \mathcal{M}_{0}$ such that

$$
\eta\left(\mu_{1}, v_{0}\right)=\eta(\mathcal{M}, \mathcal{N})
$$

If $v_{0} \in \Gamma \mu_{1}$, then

$$
\eta(\mathcal{M}, \mathcal{N}) \leq \Delta\left(\mu_{1}, \Gamma \mu_{1}\right) \leq \eta\left(\mu_{1}, v_{0}\right)=\eta(\mathcal{M}, \mathcal{N}) .
$$

Thus $\eta(\mathcal{M}, \mathcal{N})=\Delta\left(\mu_{1}, \Gamma \mu_{1}\right)$, i.e., $\mu_{1}$ is a BPP of $\Gamma$. Therefore, assume that $v_{0} \notin \Gamma \mu_{1}$. Since $\Gamma \mu_{1}$ is compact, by Lemma 2, there exists $v_{1} \in \Gamma \mu_{1}$ such that

$$
0<\eta\left(v_{0}, v_{1}\right) \leq \mathcal{H}\left(\Gamma \mu_{0}, \Gamma \mu_{1}\right) .
$$

Since $F$ is strictly increasing, the last inequality implies that

$$
\begin{aligned}
F\left(\eta\left(v_{0}, \nu_{1}\right)\right) & \leq F\left(\mathcal{H}\left(\Gamma \mu_{0}, \Gamma \mu_{1}\right)\right) \\
& \leq F\left(\eta\left(\mu_{0}, \mu_{1}\right)\right)-\tau .
\end{aligned}
$$

Since $\nu_{1} \in \Gamma \mu_{1} \subseteq \mathcal{N}_{0}$, there exists $\mu_{2} \in \mathcal{M}_{0}$ such that

$$
\eta\left(\mu_{2}, v_{1}\right)=\eta(\mathcal{M}, \mathcal{N})
$$

From (2.1) and (2.3) and using weak $P$-property, we have that

$$
\eta\left(\mu_{1}, \mu_{2}\right) \leq \eta\left(v_{0}, v_{1}\right)
$$

From (2.2) and (2.4), we have

$$
F\left(\eta\left(\mu_{1}, \mu_{2}\right)\right) \leq F\left(\eta\left(v_{0}, v_{1}\right)\right) \leq F\left(\eta\left(\mu_{0}, \mu_{1}\right)\right)-\tau .
$$

If $v_{1} \in \Gamma \mu_{2}$, then

$$
\eta(\mathcal{M}, \mathcal{N}) \leq \Delta\left(\mu_{2}, \Gamma \mu_{2}\right) \leq \eta\left(\mu_{2}, v_{1}\right)=\eta(\mathcal{M}, \mathcal{N}) .
$$

Thus $\eta(\mathcal{M}, \mathcal{N})=\Delta\left(\mu_{2}, \Gamma \mu_{2}\right)$, i.e., $\mu_{1}$ is a BPP of $\Gamma$. So, assume that $v_{1} \notin \Gamma \mu_{2}$. 
Since $\Gamma \mu_{2}$ is compact, by Lemma 2, there exists $\nu_{2} \in \Gamma \mu_{2}$ such that

$$
0<\eta\left(v_{1}, v_{2}\right) \leq \mathcal{H}\left(\Gamma \mu_{1}, \Gamma \mu_{2}\right) .
$$

Using the fact that $F$ is strictly increasing, we have that

$$
\begin{aligned}
F\left(\eta\left(v_{1}, \nu_{2}\right)\right) & \leq F\left(\mathcal{H}\left(\Gamma \mu_{1}, \Gamma \mu_{2}\right)\right) \\
& \leq F\left(\eta\left(\mu_{1}, \mu_{2}\right)\right)-\tau \\
& \leq F\left(\eta\left(\mu_{0}, \mu_{1}\right)\right)-2 \tau(\text { using } 2.5) .
\end{aligned}
$$

Since $\nu_{2} \in \Gamma \mu_{2} \subseteq \mathcal{N}_{0}$, there exists $\mu_{3} \in \mathcal{M}_{0}$ such that

$$
\eta\left(\mu_{3}, v_{2}\right)=\eta(\mathcal{M}, \mathcal{N})
$$

From (2.5) and (2.6) and using weak property $P$, we have that

$$
\eta\left(\mu_{2}, \mu_{3}\right) \leq \eta\left(v_{1}, v_{2}\right)
$$

From (2.6) and (2.7), we have

$$
F\left(\eta\left(\mu_{2}, \mu_{3}\right)\right) \leq F\left(\eta\left(v_{1}, \nu_{2}\right)\right) \leq F\left(\eta\left(\mu_{0}, \mu_{1}\right)\right)-2 \tau .
$$

Continuing in this manner, we obtain two sequences $\left\{\mu_{n}\right\}$ and $\left\{v_{n}\right\}$ in $\mathcal{M}_{0}$ and $\mathcal{N}_{0}$ respectively, satisfying

(B1) $v_{n} \in \Gamma \mu_{n} \subseteq \mathcal{N}_{0}$,

(B2) $\eta\left(\mu_{n+1}, v_{n}\right)=\eta(\mathcal{M}, \mathcal{N})$,

(B3) $F\left(\eta\left(\mu_{n}, \mu_{n+1}\right)\right) \leq F\left(\eta\left(v_{n-1}, v_{n}\right)\right) \leq F\left(\eta\left(\mu_{0}, \mu_{1}\right)\right)-n \tau$,

for each $n=0,1,2, \ldots$.

Put $\alpha_{n}=\eta\left(\mu_{n}, \mu_{n+1}\right)$ for each $n=0,1,2, \ldots$. Taking limit on both sides of (B3) as $n \rightarrow \infty$, we have

$$
\lim _{n \rightarrow \infty} F\left(\alpha_{n}\right)=-\infty
$$

Using (F2), we obtain

$$
\lim _{n \rightarrow \infty} \alpha_{n}=0 .
$$

Using (F3), there exists $k \in(0,1)$ such that

$$
\alpha_{n}^{k} F\left(\alpha_{n}\right) \rightarrow 0 \text { as } n \rightarrow \infty .
$$

From (B3), for each $n \in \mathbb{N}$, we have that

$$
F\left(\alpha_{n}\right)-F\left(\alpha_{0}\right) \leq-n \tau .
$$

This implies

$$
\alpha_{n}^{k} F\left(\alpha_{n}\right)-\alpha_{n}^{k} F\left(\alpha_{0}\right) \leq-n \alpha_{n}^{k} \tau \leq 0 .
$$

Letting $n \rightarrow \infty$ in (2.11) and using (2.9), (2.10), we obtain

$$
\lim _{n \rightarrow \infty} n \alpha_{n}^{k}=0 \text {. }
$$

Thus there exists $n_{0} \in \mathbb{N}$ such that $n \alpha_{n}^{k} \leq 1$ for all $n \geq n_{0}$, i.e., $\alpha_{n} \leq \frac{1}{n^{\frac{1}{k}}}$ for all $n \geq n_{0}$. 
Let $m, n \in \mathbb{N}$ with $m>n \geq n_{0}$. Then

$$
\begin{aligned}
\eta\left(\mu_{m}, \mu_{n}\right) & \leq \sum_{i=n}^{m-1} \eta\left(\mu_{i}, \mu_{i+1}\right)=\sum_{i=n}^{m-1} \alpha_{i} \\
& \leq \sum_{i=n}^{\infty} \alpha_{i} \leq \sum_{i=n}^{\infty} \frac{1}{i^{\frac{1}{k}}} .
\end{aligned}
$$

Since the series $\sum_{i=n}^{\infty} \frac{1}{i^{\frac{1}{k}}}$ is convergent for $k \in(0,1)$, we have $\eta\left(\mu_{m}, \mu_{n}\right) \rightarrow 0$ as $m, n \rightarrow \infty$. Hence $\left\{\mu_{n}\right\}$ is Cauchy in $\mathcal{M}_{0} \subseteq \mathcal{M}$. Since $(\Omega, \eta)$ is complete and $\mathcal{M}$ is closed, we have $\lim _{n \rightarrow \infty} \mu_{n}=\theta$ for some $\theta \in \mathcal{M}$.

Since $\Gamma$ is $\mathcal{H}$-continuous (for it is an MVFC), we have

$$
\lim _{n \rightarrow \infty} \mathcal{H}\left(\Gamma \mu_{n}, \Gamma \theta\right)=0 .
$$

Exactly in the similar manner as above, using (B3), we can prove that $\left\{v_{n}\right\}$ is Cauchy in $\mathcal{N}$ and since $\mathcal{N}$ is closed, there exists $\xi \in B$ such that $\lim _{n \rightarrow \infty} \mathcal{v}_{n}=\xi$.

Since $\eta\left(\mu_{n+1}, v_{n}\right)=\eta(\mathcal{M}, \mathcal{N})$ for all $n \in \mathbb{N}$, we have

$$
\lim _{n \rightarrow \infty} \eta\left(\mu_{n+1}, v_{n}\right)=\eta(\theta, \xi)=\eta(\mathcal{M}, \mathcal{N}) \text {. }
$$

We claim that $\xi \in \Gamma \theta$. Indeed, since $\nu_{n} \in \Gamma \mu_{n}$ for all $n \in \mathbb{N}$, we have

$$
\lim _{n \rightarrow \infty} \Delta\left(v_{n}, \Gamma \theta\right) \leq \lim _{n \rightarrow \infty} \mathcal{H}\left(\Gamma \mu_{n}, \Gamma \theta\right)=0 .
$$

Therefore, $\Delta(\xi, \Gamma \theta)=0$. Since $\Gamma \theta$ is closed, we have $\xi \in \Gamma \theta$.

Now,

$$
\eta(\mathcal{M}, \mathcal{N}) \leq \Delta(\theta, \Gamma \theta) \leq \eta(\theta, \xi)=\eta(\mathcal{M}, \mathcal{N}) .
$$

Hence $\Delta(\theta, \Gamma \theta)=\eta(\mathcal{M}, \mathcal{N})$, i.e., $\theta$ is a BPP of $\Gamma$.

A Geraghty type [8] result follows as a consequence of our previous theorem. Let $\mathcal{G}$ be the class of functions $g:[0, \infty) \rightarrow[0,1)$ satisfying the condition: $g\left(\xi_{n}\right) \rightarrow 1$ implies $\xi_{n} \rightarrow 0$. An example of such a map is $g(\xi)=(1+\xi)^{-1}$ for all $\xi>0$ and $g(0) \in[0,1)$.

Definition 7. Let $\mathcal{M}, \mathcal{N}$ be two non-empty subsets of a MS $(\Omega, \eta)$. A multivalued map $\Gamma: \mathcal{M} \rightarrow C B(\mathcal{N})$ is said to be a multivalued Geraghty-type $F$-contraction (MVGFC, in short) if there exist $\tau>0, F \in \mathcal{F}$ and $g \in \mathcal{G}$ such that

$$
\tau+F(\mathcal{H}(\Gamma \mu, \Gamma v)) \leq g(\eta(\mu, v)) \cdot F(\eta(\mu, \nu))
$$

for all $\mu, \nu \in \Omega$ with $\Gamma \mu \neq \Gamma$ v.

Corollary 1. Let $(\Omega, \eta)$ be a complete $M S$ and $\mathcal{M}, \mathcal{N}$ be two non-empty closed subsets of $\Omega$ such that $\mathcal{M}_{0} \neq \phi$ and that the pair $(\mathcal{M}, \mathcal{N})$ satisfies the weak P-property. Suppose $\Gamma: \mathcal{M} \rightarrow C B(\mathcal{N})$ be a MVGFC such that $\Gamma \mu$ is compact for each $\mu \in \mathcal{M}$ and $\Gamma \mu \subseteq \mathcal{N}_{0}$ for all $\mu \in \mathcal{M}_{0}$. Then $\Gamma$ has a BPP. 
Proof. Since $g(t) \in[0,1)$ for all $t \in[0, \infty)$, from (2.13), we have that

$$
\tau+F(\mathcal{H}(\Gamma \mu, \Gamma v)) \leq F(\eta(\mu, v))
$$

for all $\mu, v \in \mathcal{M}$ with $\Gamma \mu \neq \Gamma v$. Thus, $\Gamma$ is an MVFC and hence from Theorem 1 it follows that $\Gamma$ has a BPP.

Remark 4. Corollary 1 extends the results due to Caballero et al. [3] and Zhang et al. [18] to their multivalued analogues using $F$-contraction.

Next, we provide some examples in support of our main result.

Example 1. Consider $\Omega=\mathbb{R}$ with usual metric $\eta(\mu, v)=|\mu-v|$ for all $\mu, v \in \Omega$. Let $\mathcal{M}=[5,6]$ and $\mathcal{N}=[-6,-5]$. Then $\eta(\mathcal{M}, \mathcal{N})=10$ and $\mathcal{M}_{0}=\{5\}, \mathcal{N}_{0}=\{-5\}$. Define the multivalued map $\Gamma: \mathcal{M} \rightarrow C B(\mathcal{N})$ such that

$$
\Gamma \mu=\left[\frac{-\mu-5}{2},-5\right] \text { for all } \mu \in[5,6] .
$$

Therefore $\Gamma(5)=\{-5\}$ (i.e., $\Gamma \mu \subseteq \mathcal{N}_{0}$ for all $\mu \in \mathcal{M}_{0}$ ).

We claim that $\Gamma$ is a MVFC. Let $\mathcal{H}(\Gamma \mu, \Gamma v)>0$. Then we have

$$
\begin{aligned}
\mathcal{H}(\Gamma \mu, \Gamma v) & =\mathcal{H}\left(\left[\frac{-\mu-5}{2},-5\right],\left[\frac{-v-5}{2},-5\right]\right) \\
& =\left|\left(\frac{-\mu-5}{2}\right)-\left(\frac{-v-5}{2}\right)\right| \\
& =\frac{|v-\mu|}{2} \\
& =\frac{\eta(\mu, v)}{2} \\
& <\eta(\mu, v) .
\end{aligned}
$$

From the last inequality, we have that $\ln (\mathcal{H}(\Gamma \mu, \Gamma v))<\ln (\eta(\mu, v))$, and further, $\tau+\ln (\mathcal{H}(\Gamma \mu, \Gamma v)) \leq \ln (\eta(\mu, v))$, for any $\tau \in(0, \ln 2]$. Therefore, we have that $\tau+F(\mathcal{H}(\Gamma \mu, \Gamma v)) \leq F(\eta(\mu, v))$, for any $\tau \in(0, \ln 2]$, where $F(t)=\ln t, t>0$.

Finally, it is easy to check that $(\mathcal{M}, \mathcal{N})$ satisfies weak $P$-property. Thus, all conditions of Theorem 1 are satisfied and we observe that $\mu=5$ is a BPP of $\Gamma$.

In fact, in Example 1, the pair $(\mathcal{M}, \mathcal{N})$ satisfies $P$-property (and hence the weak $P$-property as well). Next, we present an example in which the pair $(\mathcal{M}, \mathcal{N})$ satisfies only the weak $P$-property but not the $P$-property.

Example 2. Consider $\Omega=\mathbb{R}^{2}$ with the Euclidean metric $\eta$.

Let $\mathcal{M}=\{(-5,0),(0,1),(5,0)\}$ and $\mathcal{N}=\left\{(\mu, v): v=2+\sqrt{2-\mu^{2}}, \mu \in[-\sqrt{2}, \sqrt{2}]\right\}$. Then $\eta(\mathcal{M}, \mathcal{N})=\sqrt{3}$ and $\mathcal{M}_{0}=\{(0,1)\}, \mathcal{N}_{0}=\{(\sqrt{2}, 2),(-\sqrt{2}, 2)\}$.

Define the multivalued map $\Gamma: \mathcal{M} \rightarrow C B(\mathcal{N})$ such that

$$
\Gamma(-5,0)=\{(-\sqrt{2}, 2),(-1,3)\}, \Gamma(0,1)=\{(\sqrt{2}, 2)\}, \Gamma(5,0)=\{(\sqrt{2}, 2),(1,3)\} .
$$


It is easy to check that $\Gamma$ is a MVFC with $\tau=\ln 2$ and $F(t)=\ln t, t>0$.

Finally, we observe that

$$
\eta((0,1),(\sqrt{2}, 2))=\eta((0,1),(-\sqrt{2}, 2))=\sqrt{3}=\eta(\mathcal{M}, \mathcal{N}),
$$

but

$$
\eta((0,1),(0,1))=0<\eta((\sqrt{2}, 2),(-\sqrt{2}, 2))=2 \sqrt{2} .
$$

Thus, $(\mathcal{M}, \mathcal{N})$ satisfies weak $P$-property, but not the $P$-property. Therefore, all conditions of Theorem 1 are satisfied and since $\Delta((0,1), \Gamma(0,1))=\sqrt{3}=\eta(\mathcal{M}, \mathcal{N})$, we conclude that $(0,1)$ is a BPP of $\Gamma$.

\section{CONCLUSION}

We have proved our main result with a strong condition that images of the MVFC are compact sets. Relaxation of this compactness criterion is a suggested future work. We have shown the non-triviality of the assumption of the weak $P$-property by presenting an example which does not satisfy the $P$-property but satisfies only the weak $P$-property. The results due to Caballero et al. [3] and Zhang et al. [18] are also extended to their multivalued analogues as a consequence of our results.

\section{ACKNOWLEDGEMENT}

The author expresses his hearty gratitude to the learned referees for their constructive comments which have improved the manuscript considerably.

\section{REFERENCES}

[1] I. Altun, G. Minak, and H. Dag, "Multivalued $F$-contractions on complete metric spaces," $J$. Nonlinear Convex Anal., vol. 16, no. 4, pp. 659-666, 2015.

[2] M. Boriceanu, A. Petrusel, and I. Rus, "Fixed point theorems for some multivalued generalized contractions in b-metric spaces," Internat. J. Math. Statistics, vol. 6, pp. 65-76, 2010.

[3] J. Caballero, J. Harjani, and K. Sadarangani, "A best proximity point theorem for Geraghtycontractions," Fixed Point Theory Appl., vol. 2012, no. 231, pp. 1-9, 2012, doi: 10.1186/16871812-2012-231.

[4] S. Czerwik, "Nonlinear set-valued contraction mappings in b-metric spaces," Atti Sem. Mat. Univ. Modena, vol. 46, pp. 263-276, 1998.

[5] P. Debnath and M. de La Sen, "Fixed points of eventually $\Delta$-restrictive and $\Delta(\varepsilon)$-restrictive set-valued maps in metric spaces," Symmetry, vol. 12, no. 1, pp. 1-7, 2020, doi: 10.3390/sym12010127.

[6] P. Debnath and H. M. Srivastava, "Global optimization and common best proximity points for some multivalued contractive pairs of mappings," Axioms, vol. 9, no. 3, pp. 1-8, 2020, doi: 10.3390/axioms9030102.

[7] P. Debnath and H. M. Srivastava, "New extensions of Kannan's and Reich's fixed point theorems for multivalued maps using Wardowski's technique with application to integral equations," Symmetry, vol. 12, no. 7, pp. 1-9, 2020, doi: 10.3390/sym12071090.

[8] M. A. Geraghty, "On contractive mappings," Proc. Amer. Math. Soc., vol. 40, pp. 604-608, 1973.

[9] S. B. Nadler, "Multi-valued contraction mappings," Pac. J. Math., vol. 30, no. 2, pp. 475-488, 1969, doi: 10.2140/pjm.1969.30.475. 
[10] E. Nazari, "Best proximity point theorems for generalized multivalued contractions in metric spaces," Miskolc Math. Notes, vol. 16, no. 2, pp. 1055-1062, 2015, doi: 10.18514/MMN.2015.1329.

[11] M. Omidvari, S. M. Vaezpour, and R. Saadati, "Best proximity point theorems for Fcontractive non-self mappings," Miskolc Math. Notes, vol. 15, no. 2, pp. 615-623, 2014, doi: 10.18514/MMN.2014.1011.

[12] V. Sankar Raj, "A best proximity point theorem for weakly contractive non-self-mappings," Nonlinear Anal., vol. 74, no. 14, pp. 4804-4808, 2011, doi: 10.1016/j.na.2011.04.052.

[13] H. M. Srivastava, S. V. Bedre, S. M. Khairnar, and B. S. Desale, "Krasnosel'skii type hybrid fixed point theorems and their applications to fractional integral equations," Abstr. Appl. Anal., vol. 2014, no. Article ID: 710746, pp. 1-9, 2014, doi: 10.1155/2014/710746.

[14] H. M. Srivastava, S. V. Bedre, S. M. Khairnar, and B. S. Desale, "Corrigendum to "Krasnosel'skii type hybrid fixed point theorems and their applications to fractional integral equations"," Abstr. Appl. Anal., vol. 2015, no. Article ID: 467569, pp. 1-2, 2015, doi: 10.1155/2015/467569.

[15] H. M. Srivastava, A. Shehata, and S. I. Moustafa, "Some fixed point theorems for $f(\psi, \varphi)$ contractions and their application to fractional differential equations," Russian J. Math. Phys., vol. 27, pp. 385-398, 2020, doi: 10.1134/S1061920820030103.

[16] D. Wardowski, "Fixed points of a new type of contractive mappings in complete metric space," Fixed Point Theory Appl., vol. 2012, no. 94, pp. 1-6, 2012, doi: 10.1186/1687-1812-2012-94.

[17] Q. Xu, Y. Tang, T. Yang, and H. M. Srivastava, "Schwarz lemma involving the boundary fixed point," Fixed Point Theory Appl., vol. 2016, no. Article ID: 84, pp. 1-8, 2016, doi: 10.1186/s13663-016-0574-8.

[18] J. Zhang, Y. Su, and Q. Cheng, "A note on 'A best proximity point theorem for Geraghtycontractions'," Fixed Point Theory Appl., vol. 2013, no. 99, pp. 1-4, 2013, doi: : 10.1186/16871812-2013-83.

\section{Author's address}

\section{Pradip Debnath}

Department of Applied Science and Humanities, Assam University, Silchar, Cachar, Assam - 788011, India

E-mail address: debnath.pradipeyahoo.com 